\title{
Intensive Batch Scheduling Application for Parallel Programming In the Cloud Computing Infrastructure
}

\author{
K.M.Azaraffali ${ }^{1}$, Dr.T.Krishnakumar ${ }^{2}$, Dr.M.Sriram ${ }^{3}$ \\ \{azaraffali@gmail.com ${ }^{1}$, drkk@bharathuniv.ac.in², msr1sriram@gmail.com ${ }^{3}$ \}
}

\begin{abstract}
Research Scholar, CSE, Bharath Institute of Higher Education and Research, Chennai, India ${ }^{1}$, Professor, CSE, Bharath Institute of Higher Education and Research, Chennai, India. ${ }^{2}$, Assistant Professor, Department of CSE, Bharath Institute of Higher Education and Research, Chennai ${ }^{3}$
\end{abstract}

\begin{abstract}
Enterprises are gathering and analyzing an increasing number of large amounts of facts to be able to derive enterprise insights. Two challenges to fulfill. First, in parallel programming is together time-consuming and error-susceptible. Second, the developing Cloud Computing paradigm executes the primary infrastructure, which armies Enterprises to reconsideration their request structure. Now we advocate massive scale data-intensive batch troubles below the Cloud infrastructure restraints. A programming version and fleeces the difficulty of parallel programming, it offers the customers broad manipulate on facts separated and distribute calculation the applications. We reap high overall performance in computing Cloud and parallel programming is much less time and performance.
\end{abstract}

Keywords: Cloud Computing, Intensive Batch Scheduling, Parallel Programming.

\section{Introduction}

To remain in front of rivalry, Enterprises are gathering and investigating huge measure of information to determine business bits of knowledge, ideally continuously or close to constant. For instance, Enterprises need to comprehend their clients better to build up a superior promoting plan, or inspect their production network to search for freedoms to recover productivity, or investigate sensors information to anticipate machine disappointment and forestall income lost before [7]. 


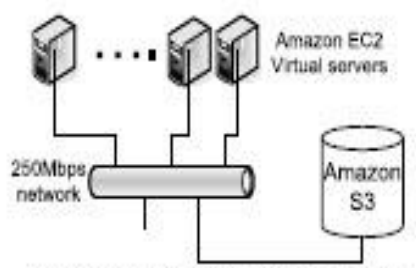

(8) Logieal view of Amazen EC2 infrastructure

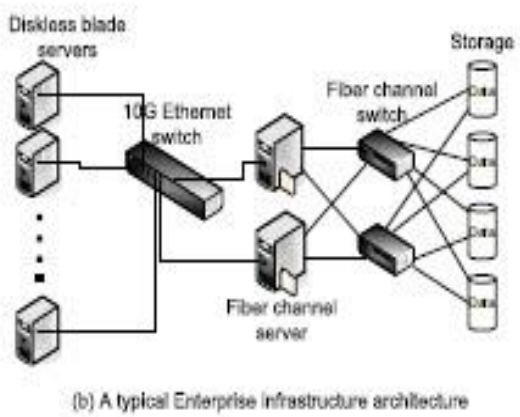

Figure 1. Comparison of infrastructure architectures

The patterns on information development and processor speed development propose that certain type of parallelization is needed to investigate the information. To begin with, because of the needs to determine improved business bits of knowledge recover exactness, measure information gathering is developing at an outstanding rate. Second, uniprocessor speed has halted remarkable development unevenly [8]. Numerous applications are as of now requiring hours to long stretches of calculation time to deal with the information nowadays, and it will revenue any lengthier after some information volume develops. handling postpones put Enterprises at serious drawbacks can't respond sufficiently quick. It is regularly equipment part disappointment and inconsistent information recuperation.

The Propose Grid Batch framework, which targets tackling huge scope information concentrated clump issues under the Haze foundation constraints[9]. GridBatch is a programming perfect and related collection that conceals the intricacy of equal software design, yet it stretches the clients unlimited oversight on how information are apportioned and how calculation is circulated so application container have the best conceivable. GridBatch accomplishes elite in Amazon's EC2 figuring Cloud. GridBatch which performs well in a Cloud framework, yet additionally makes it simple to compose equal projects for information concentrated cluster applications. A powerful urge to investigate the information in the arising Cloud Computing framework on account of its solid incentives. 


\section{Related Work}

Conventional data sets can be utilized to carry out numerous examination applications we are focusing on. Lamentably, they don't scale for huge informational collections for two reasons.

1. In the first place, information bases present a significant level SQL language fully intent on concealing the execution subtleties. Albeit simple to utilize, this general language powers clients to communicate calculation in manners that are definitely not execution proficient. Some of the time, the most effective technique would possibly filter the informational index once. Despite the fact that methods have been created to consequently upgrade inquiry handling, the exhibition is still a long way from accomplished software engineer comprehends the applications well.

2. Second, information bases route glowing in a conventional Enterprises framework engineering network transfer speed abundant, however endure seriously in a Cloud Computing foundation since can't abuse the nearby plate I/O data transmission. Despite the fact that most information base frameworks, including Oracle's business items, utilize modern storing instruments, numerous information gets to in any case navigate the organization, devouring valuable data transmission.

\section{Gridbatch System}

There are two urgent data type in Grid Batch table or documented table (procured from informational collection phrasing). A table contain a lot of records (pushes) that are liberated from each other. All records in a table follows a comparable planning, and each record may contain a couple of field (sections). Documented table resembles table beside that each record also has a connected rundown, where the rundown could basically be one of the field or other data gave by the user[10]. Table is undifferentiated from the vector thought in vector or SIMD machine or the stream thought in stream processors [7] in Computer Architecture. Basically, table grants us to design an item structure which can deal with the records in equivalent across various machines in the Cloud. The GridBatch system contains two pieces of connected programming parts: the appropriated archive structure (DFS) and the work scheduler.

\section{A The distributed file system}

DFS is an augmentation of GFS [5] that upholds another sort of record. DFS store two sorts of records: fixed-piece size documents or fixed-num-of-lump documents. For filed table, let us think about another kind of documents: fixed-num-of-piece records, where each record has a fixed number of lumps and every lump could have self-assertively enormous size[11]. At the point when another information record should be composed, the DFS customer call the parcel capacity to decide the lump number, at that point it annexes the greatest to the furthest limit of the chunk.

\section{B Job Scheduling}


The work planning framework incorporates an expert hub and numerous slave hubs. The slave hub is answerable for running an errand appointed by the expert hub. The expert hub is liable for separating a task into numerous more modest undertakings as communicated in the client programs. It disperses the undertakings crossways all slave hubs in the framework, and it screens the errands to ensure every one of them whole effectively. A slave hub is regularly an information hub. At the point when the expert timetables an undertaking, it could plan the assignment on the hub which holds the piece of information to be prepared. By preparing information on the neighborhood hub, we save money on valuable organization transmission capacity.

\section{Gridbatch Operators}

GridBatch doesn't endeavor a developer reasons the superlative way to deal with program an applications. All things being equal, it intends to give a bunch of ordinarily utilized natives, called administrators, which the software engineer money on programming endeavors. The administrators handle the subtleties of circulating various machine, in this way the client may not have to stress over equal programming. All things considered, the client simply needs to apply a bunch of administrators consecutively, similarly as though composing a customary successive program. GridBatch separates MapReduce into rudimentary administrators, and moreover, presents extra administrators. GridBatch presently comprises of the accompanying administrators:

\section{A Map Operator}

The Map operator smears a user-defined purpose finished all annals of a table.

\section{B Distribute Operator}

The Distribute administrator changes a table or a recorded table over to additional ordered table with an alternate file. The subsequent listed table is put away as a solitary fixed-num-ofpiece DFS document. This includes rearranging information from whichever lump the information was on beforehand to another piece as shown by the segment work for the new file.

\section{Join Operator}

The Join administrator takes two filed tables and union the relating record if the list field competition. The GridBatch framework tracks down the comparing record that have a coordinating with file, and afterward conjures a tradition capacity characterized by the client. The client characterized capacity can basically combine the dual record, as in a customary information base joint, or it can play out some exceptional activity as it wants.

\section{Cartesian Operator}

In contrast to the Join administrator, which possibly coordinates with records when their list field matchs, the Cartesian administrator coordinate with each records of Table $\mathrm{X}$ each record of Table $\mathrm{Y}$, and smear a client characterized work. 


\section{E Recurse Operator}

Many decrease activities are commutative and cooperative, and thus, request autonomous. For these request autonomous lessen tasks, we present the Recurse administrator.

F Neighbor Operator

Examination capacities, like our customer's interweaving discovery issue, need to dissect the grouping to determine significant outcomes. The Neighbor administrator bunches adjoining records and conjures a client characterized capacity to dissect the sub-succession.

\section{Conclusion}

Because of cutthroat pressing factor, Enterprise needs to dissect a lot of information in a short measure of time, under a clashing objective of utilizing the smallest expense conceivable. There a few difficulties in gathering these objectives. To begin with, the measure of information we gather is huge to such an extent now past ability of a uniprocessor measure in a sensible measure of time. The development pattern of information volume and uniprocessor limit recommends issue can't be settled by innovation climbing. All things being equal, some type of equal handling is essential. Sadly, composing equal projects is intrinsically troublesome because of the disseminated nature.

Second, despite the fact that Cloud Computing vows to significantly bring down the expense, particularly for time-fluctuating calculation requests, its foundation is altogether different from the customary Enterprise framework. Utilizing a genuine customer application model, we presented how these administrators might be utilized to tackle genuine issues. Finished tests, we presented that the administrators stretch the clients not just straight control on information development and capacity yet additionally adaptability in piece, so the application can accomplish better, not just contrasted with the customary methodology in a conventional Enterprise framework, yet in addition contrasted with MapReduce in a Cloud foundation

\section{References}

[1] M. Atkinson. Uk e-science grid infrastructure meets biological research challenges. http://www.nesc.ac.uk/talks/mpa/BioGridCambridge2Oct02.ppt, Oct. 2002.

[2] H. chih Yang, A. Dasdan, R.-L. Hsiao, and D. S.Parker. Map-reduce-merge: Simplified relational data processing on large clusters. In Proc. SIGMOD, 2007.

[3] T. H. Davenport and J. G. Harris. Competing on Analytics: The New Science of Winning. Harvard Business School Press, 2007.

[4] J. Dean and S. Ghemawat. Mapreduce: Simplified data processing on large clusters. In OSDI'04: Sixth Symposium on Operating System Design and Implementation, December 2004.

[5] S. Ghemawat, H. Gobioff, and S.-T. Leung. The google file system. In 19th ACM Symposium on Operating Systems Principles, October 2003.

[6] M. Isard, M. Budiu, Y. Yu, A. Birrell, and D. Fetterly. Dryad: Distributed data-parallel programs from sequential building blocks. In European Conference on Computer Systems (EuroSys), March 2007. 
[7] Dr. Kaliyamurthie K.P "An Application Of Non-Uniform Cellular Automata For Efficient Cryptography", Indian Journal of Science and Technology, Vol 6, Issue 5S, page 4648-4652 May 2013.

[8] Dr. Kaliyamurthie K.P "K-Anonymity Based Privacy Preserving For Data Collection In Wireless Sensor Networks", Indian Journal of Science and Technology, Vol 6, Issue 5S, page 4604-4614 May 2013.

[9] Dr. Kaliyamurthie K.P "Highly Secured Online Voting System Over Network" , Indian Journal of Science and Technology, Vol 6, Issue 6S page 4831-4836 May 2013.

[10] Dr. Kumaravel. A "Vehnode: Wireless Sensor Network Platform For Automobile Pollution Control" IEEE explore, Vol Page(s): 963 - 966, 2013.

[11] Dr. Kumaravel. A "Multi- Classification Approach For Detecting Network" IEEE explore, Page(s): 1114 -1117,April 2013.

[12] T. Vijayan, M. Sangeetha, A. Kumaravel \& B. Karthik (2020): FeatureSelection for Simple Color Histogram Filter based on Retinal Fundus Images for DiabeticRetinopathy Recognition, IETE Journal of Research, DOI: 10.1080/03772063.2020.1844082.

[13] D. S. Vijayan, A. Leema Rose, S. Arvindan, J. Revathy, C. Amuthadevi, “Automation systems in smart buildings: a review", Journal of Ambient Intelligence and Humanized Computing https://doi.org/10.1007/s12652-020-02666-9

[14] Vijayan T, Sangeetha M, A. Kumaravel, Karthik B, "Gabor filter and machine learning based diabetic retinopathy analysis and detection", Microprocessors and Microsystems,2020. https://doi.org/10.1016/j.micpro.2020.103353.

[15] Vijayan T, SangeethaM, Karthik B, "Trainable WEKA Segmentation of Retinal Fundus Images for Global Eye Disease Diagnosis Application," International Journal of Emerging Trends in Engineering Research,Vol 8, No.9, pp. 5750-5754, Sep 2020. https://doi.org/10.30534/ijeter/2020/136892020

[16] C. Amuthadevi, D. S. Vijayan, Varatharajan Ramachandran, "Development of air quality monitoring (AQM) models using different machine learning approaches", Journal of Ambient Intelligence and Humanized Computing, https://doi.org/10.1007/s12652-020-02724-2

[17] Vijayan T, Sangeetha M, A. Kumaravel, Karthik B, "Fine Tuned VGG19 Convolutional Neural Network Architecture for Diabetic Retinopathy Diagnosis," Indian Journal of Computer Science and Engineering (IJCSE), Vol. 11, No. 5, pp. 615-622 Sep-Oct 2020. DOI: 10.21817/indjcse/2020/v11i5/201105266.

[18] Vijayan T, Sangeetha M, Karthik B, "Efficient Analysis of Diabetic Retinopathy on Retinal Fundus Images using Deep Learning Techniques with Inception V3 Architecture," Journal of Green Engineering, Vol 10, Issue 10, pp. 9615-9625. Oct 2020 